Document downloaded from:

http://hdl.handle.net/10251/61581

This paper must be cited as:

Cuesta Frau, D.; M. Varela-Entrecanadles; R. Valor-Pérez; B. Vargas (2015). Development of a novel scheme for long-term body temperature monitoring: a review of benefits and applications. Journal of Medical Systems. 4(39):1-7. doi:10.1007/s10916-015-0209-3.

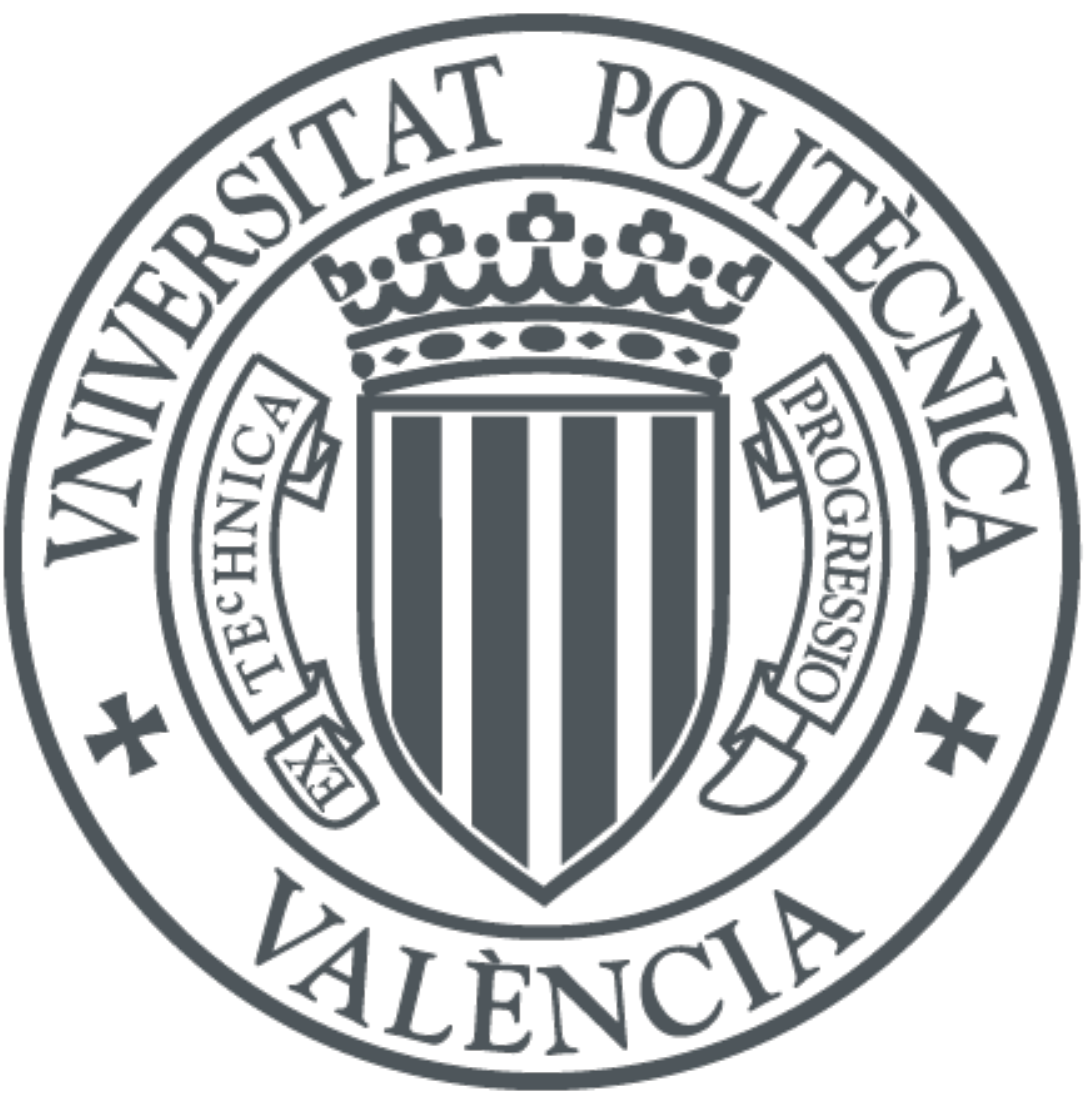

The final publication is available at

http://dx.doi.org/10.1007/s10916-015-0209-3

Copyright Springer Verlag (Germany)

Additional Information 


\title{
Development of a Novel Scheme for Long-term Body Temperature Monitoring: A Review of Benefits and Applications
}

\author{
David Cuesta-Frau · Manuel \\ Varela-Entrecanales · Raul Valor-Perez • \\ Borja Vargas
}

Received: date / Accepted: date

\begin{abstract}
Body temperature is a health or disease marker that has been in clinical use for centuries. The threshold currently applied to define fever, with small variations, is $38{ }^{\circ} \mathrm{C}$. However, current approaches do not provide a full picture of the thermoregulation process and its correlation with disease.

This paper describes a new non-invasive body temperature device that improves the understanding of the pathophysiology of diseases by integrating a variety of temperature data from different body locations. This device enables to gain a deeper insight into fever, endogenous rhythms, subject activity and ambient temperature to provide anticipatory and more efficient treatments. Its clinical use would be a big step in the overcoming of the anachronistic febrile/afebrile dichotomy and walking towards a system medicine approach to certain diseases.

This device has already been used in some clinical applications successfully. Other possible applications based on the device features and clinical requirements are also described in this paper.
\end{abstract}

Keywords Body temperature · Continuous monitoring · Fever · medical diagnosis $\cdot$ nursing $\cdot$ thermometer

David Cuesta-Frau

Technological Institute of Informatics, Polytechnic University of Valencia, Alcoi Campus, Plaza Ferrandiz y Carbonell, 2, 03801 Alcoi (Spain)

Tel.: +34-651028565

E-mail: dcuesta@disca.upv.es

Manuel Varela-Entrecanales

Department of Internal Medicine, Teaching Hospital of Mostoles, Madrid (Spain)

Raul Valor-Perez

Innovatec Sensing and Communications, Avda Elx, 03801 Alcoi (Spain)

Borja Vargas

Biomedical Science Faculty, European University of Madrid, Villaviciosa de Odon, 28670 Madrid (Spain) 


\section{Introduction}

Continuous monitoring of certain clinical variables, either at bedside or ambulatory, provides important information about the physiological and pathophysiological state of the patient that would otherwise not be accessible $[1,2]$. Furthermore, in contrast to intermittent monitoring, continuous monitoring can have a positive influence on the prognosis and outcomes $[3,4]$. This could also be the case with body temperature, one of the four main vital signs.

Temperature monitoring in medicine provides important data required to guide delivery of care [5]. It is a perfectly established clinical variable, whose importance needs not be emphasized. It is the core temperature that clinicians are usually interested in. Measurement of core body temperature requires the placement of a pulmonary artery catheter. Alternatives include invasive temperature monitoring (esophageal, bladder, rectal) or noninvasive methods (oral, earbased, temporal artery, or axillary). Clinicians are challenged to select the measurement method that is most appropriate for a patient and provides the most accurate and precise approximation of core temperature.

Body temperature is considered a vital constant. Its elevation (fever) is a well-recognized symptom of disease and it is routinely monitored in patients admitted to hospital. It is normal practice to classify patients as febrile or afebrile, according to a fixed and arbitrary red line $\left(38^{\circ} \mathrm{C}\right)$. However, this is probably a gross oversimplification [9]. Temperature is not a constant, and it is not homogeneous throughout the body. It is probably better conceived as a dynamic equilibrium state between heat production and heat dissipation, delicately fine-tuned by means of several interwoven heat-conserving and heatdissipating mechanisms. Thermoregulation is arguably a prototypical example of a complex dynamic system, harbouring some of its remarkable properties (resilience, development of spontaneous rhythms, presence of strange attractors). Continuous monitoring and analysis of central and peripheral temperature would detect the building of a fever, therefore allowing its forecast before it appears. Blood cultures drawn at this moment should have considerably higher yield than those drawn conventionally.

However, in daily practice, the use of body temperature is hampered by several misconceptions that arguably impairs its full clinical performance:

- It is considered as a "vital constant", and thus sampled at a very low frequency (e.g. every 6 - 8 hours).

- It is considered a "state" rather than a process.

- It is usually considered as a dichotomic variable (febrile/afebrile).

While research studies have shown the usefulness of the temperature timeseries for patient assessment and its prognostic value has been documented [11], the use of high-frequency/high accuracy temperature time-series and associated analysis metrics have not been extended to clinical environments due to the lack of commercial temperature monitoring systems with adequate sampling frequency, resolution, and analysis capabilities. Consequently, even though thermal physiology has undergone big conceptual and technical 
changes, clinical thermometry has remained a rather stagnant discipline, and its conceptual framework has also remained static for the last decades due to the lack of adequate monitoring systems.

This paper describes a new clinical temperature data recorder that brings into general practice the novel and full of potential application of this biosignal as a continuous variable instead. Body temperature is a quantitative, continuous variable. However, it is always used as a qualitative, flip-flop variable. Once fever has been confirmed, it is assumed that the patient is sick, but nothing can be said about the fever aetiology. Classic fever patterns have almost uniformly fallen in discredit and clinicians do not usually pay attention to temperature profiles. Even in acute care settings, temperature time series are generally sampled at a very low frequency, at a single site and only conventional statistics are usually applied. The new body temperature device addressed in this paper is devised to become a novel diagnostic, susceptibility/risk, monitoring, and/or prognostic tool that could have a high potential for short term uptake into clinical practice.

\section{Device description}

We propose the creation of a device that accurately measures body temperature long term in an ambulatory fashion using up to 4 channels simultaneously. This device is based on that described in [10], and tested in [11], but adding the following capabilities:

- Measure simultaneously temperature at 4 channels/locations. This enables the parallel monitoring of thermoregulation and perfusion at different sites. Specifically, the device includes two channels with measurement range from $31{ }^{\circ} \mathrm{C}$ to $41^{\circ} \mathrm{C}$, to measure body temperature at two locations. One channel with measurement range from $19^{\circ} \mathrm{C}$ to $41^{\circ} \mathrm{C}$, to measure temperatures during hypothermia, or to serve as an additional body site, and a fourth channel to measure from $-10{ }^{\circ} \mathrm{C}$ up to $50{ }^{\circ} \mathrm{C}$, to account for ambient temperature or another body temperature channel.

- Include a display to show temperature values and enable a basic interaction with users by means of a configuration menu.

- Measure subject actigraphy using a 3D accelerometer. This enables the correlation of body temperature with physical activity and therefore filter out data that do not correspond to pathology information.

- Include a self-calibration stage. The device contains a set of reference points of high accuracy and low variance that enables in-house accuracy checks and offset correction.

The device can use different temperature probes such as skin, tympanic, rectal, urethral, or ambient temperature sensors, either reusable or disposable. Sampling rate is user selectable, from 30s up to 15 minutes, in 30s steps. Data can be transmitted directly to a local recipient or remotely over a communication network. Temperature readings may be displayed on the device or using a 


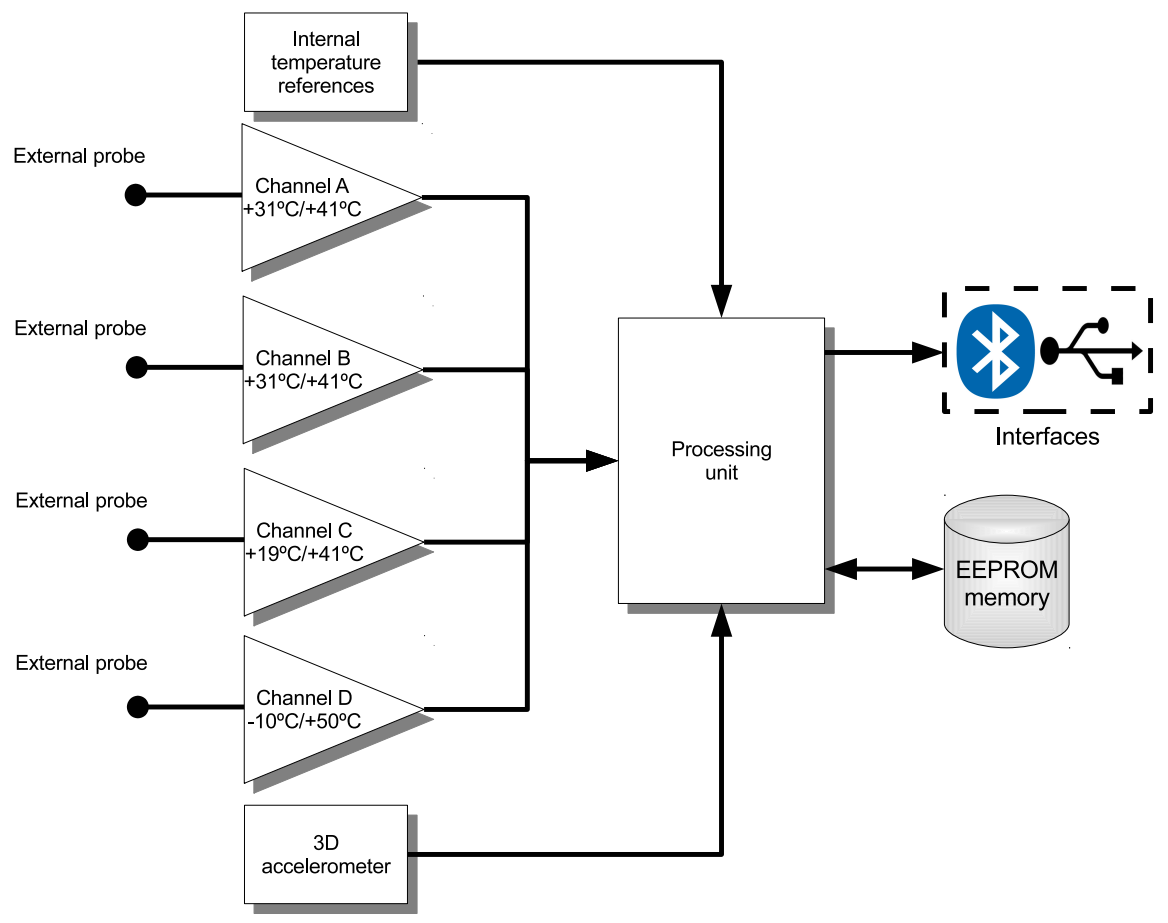

Fig. 1 Block diagram of the body temperature measurement device proposed. Data are collected simultaneously from the 4 channels, including actigraphy information. All the data are stored and transmitted using a Bluetooth wireless interface or a USB connection.

data receiver (personal computer, smartphone, tablet), using the corresponding software tool. This tool can also be configured for remote alarm reporting. The main blocks of the device are shown in Fig. 1.

The temperature probes can be attached to the patient at different locations as stated above, depending on the probe type. The device can then be configured in terms of sampling rate, communication interface (wireless Bluetooth or wired USB), and acquisition mode: real-time or off-line Holter mode. Henceforth, temperature and actigraphy data are collected, stored, and transmitted to a host computer or visualization device. A local memory is included to store data and avoid missing samples due to out of range communications or interferences. As a portable device, it includes a battery module to power the system up to 14 days.

There are many other systems for body temperature measurement. However, no device currently matches the capabilities of the one described in this paper. In Table 1, a comparison is performed among the most common approaches in clinical settings. 


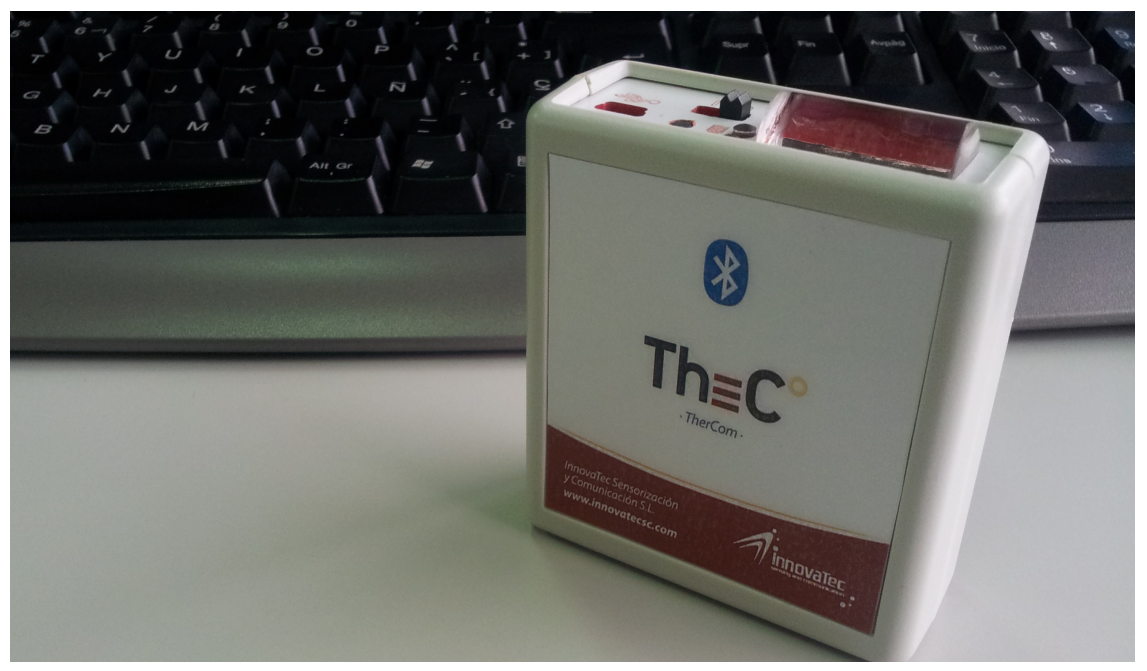

Fig. 2 Image of the actual device. Probes are connected using the sockets at the bottom of the device. At the top, the display, on-off switch, USB connector, LEDs, and interaction button are located.

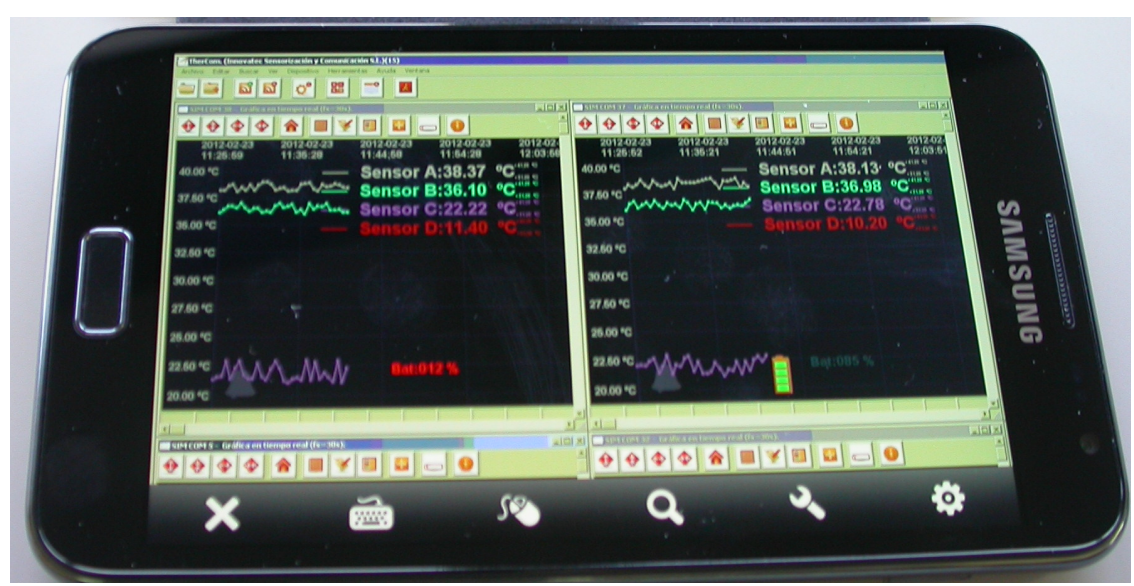

Fig. 3 Example of temperature data visualization on a smartphone. There are two devices simultaneously collecting temperature data from 4 channels, not only body measurements, but also indoor and outdoor data. 
Table 1 Comparison of some of the most popular devices for body temperature measurement in clinical settings. Desired features are in bold.

\begin{tabular}{|c|c|c|c|c|c|c|}
\hline & $\begin{array}{l}\text { Pulmonary, } \\
\text { Esophageal, } \\
\text { Bladder }\end{array}$ & Mercury & Chemical & Infrared & $\begin{array}{l}\text { Internal } \\
\text { pill }\end{array}$ & Proposed \\
\hline High accuracy & Yes & No & No & No & Yes & Yes \\
\hline Injury risk & Yes & No & No & No & No & No \\
\hline Portable & No & No & No & No & Yes & Yes \\
\hline Low-cost & No & Yes & Yes & Yes & No & Yes \\
\hline Multichannel & No & No & No & No & No & Yes \\
\hline Actigraphy & No & No & No & No & No & Yes \\
\hline $\begin{array}{l}\text { Temperature } \\
\text { record }\end{array}$ & Yes & No & No & No & Yes & Yes \\
\hline $\begin{array}{l}\text { Patient } \\
\text { convenience }\end{array}$ & No & Yes & Yes & Yes & No & Yes \\
\hline $\begin{array}{l}\text { Information } \\
\text { integration }\end{array}$ & No & No & No & No & No & Yes \\
\hline
\end{tabular}

\section{Testing and Results}

This device, including simplified versions, has been tested in actual clinical settings. The rationale of these approaches is that temperature monitoring is addressed using a long-term scheme and at a high sampling rate, in a similar way as Holter monitoring of cardiac activity, in contrast to low-frequency, isolated measurements. Some of these tests are reported in Sections 3.1-3.4.

\subsection{Fever peak detection}

Many fever peaks can be overlooked in a traditional body temperature measurement procedure, since three-times or four-times a day measurements leave too long unattended time periods. This issue was studied in [6]. The two main findings of this study were that conventional temperature monitoring overlooks a significant proportion of fever peaks (the new method found a mean of 0.73 more peaks than low frequency readings), and, in addition, a high-frequency sampling of temperature such as the one proposed in this paper, may reveal some otherwise hidden characteristics of thermoregulation, such as differences between tuberculosis and HIV patients.

\subsection{Correlation with SOFA score}

The SOFA score (Sequential Organ Failure Assessment) has been demonstrated as a good indicator of prognosis [7]. It is one of the most used predictive scoring systems in intensive care units [8]. These scoring systems are based on a number of weighted quantifiable physiological parameters. 
On the other hand, we have observed that there is a progressive loss of complexity from a normality state to illness in thermo-regulation and in glucoregulation, and such loss entails a worse prognosis. There is an inverse correlation between variability and complexity, and probably both phenomena are manifestations of the same deterioration process of the fine control physiological systems. Based on this observation, we studied if a complexity analysis of body temperature could offer methods much more objective, sensitive, and accurate to quantify such deterioration, and therefore, correlate it with classical scoring systems such as SOFA. The results of this study were reported in [17]. A significant correlation with temperature complexity and SOFA score was found in $89 \%$ of the patients. Temperature complexity, in contrast to scoring indices, is non-invasive, enables immediate computation, it only needs a single parameter, and it is inexpensive and convenient for both patients and physicians.

\subsection{Predicting survival in critical patients}

Using body temperature entropy estimation, in a similar way as in Sec.3.2, we found a correlation between a patient condition, and their body temperature regularity $[18,19]$. There was a statistically significant difference between the regularity of signals from patients that survived and patients that did not $(72 \%$ classifier accuracy). In order to use this methodology, the body temperature sampling frequency must be relatively high compared to the classical schemes, and long-term, several days or even weeks, as provided by the device proposed in this paper.

\subsection{Pre-febrile profile}

Fever does not appear suddenly. The final temperature rise is just the summit of a long process that involves several stages. A central mechanism in temperature control is the fine regulation of the amount of blood circulating through peripheral capillary networks vs. the amount of blood being by-passed to deeper (and more insulated) circuits. This can be evaluated through simultaneous monitoring of central and peripheral temperature. In a preliminary study on patients admitted to a general internal medicine ward, we developed a logistic regression model that could predict the appearance of a fever spike in the following 15-60 minutes with a sensitivity of 0.83 and a specificity of 0.85 (area under the ROC curve: 0.91)[12]. The variables selected by the logistic model were the core and peripheral temperature, their gradient and correlation during the last hour, the correlation between gradient and core temperature during the last hour, change in this correlation in the last 30 minutes and the Approximate Entropy [13] of the core temperature in the last two hours. This model was validated with a new sample. The sensitivity and specificity to predict the development of fever in the following 15-120 minutes 
was 0.67 and 0.9 respectively, with an area under the ROC curve of 0.83 [12]. Forecasting fever could eventually allow for an early intervention, avoiding its development in certain patients (e.g. infants or frail elderly, haemodynamically unstable patients, etc.).

\subsection{Other applications}

This device provides a new tool that opens the door to a number of advanced clinical applications. Some of these applications could be:

- Immunocompromised patients. This patients are predisposed to infections that may result in fever [14]. Fever is an extremely important clinical sign that should prompt immediate consultation, and a major factor regarding prognosis is the time delay until the initiation of antibiotics.

- Neurogenic fever. This fever is normally seen in patients after suffering traumatic brain injury. The usual therapy is to reduce the temperature of the injured brain, which requires a continuous and accurate temperature monitoring, and real time assessment of the hyperthermia management protocols.

- Hypothermia. A number of clinical conditions can lead to hypothermia: diabetes, heart failure, trauma, kidney disease, or patients in a frailty state. As in many medical applications, an early intervention can have a great impact on prognosis.

- Fever of unknown origin. These fevers correspond to cases where, after at least one week of study, it is not possible to determine the cause of fever. These patients require a continuous ambulatory body temperature monitoring for at least three weeks.

- General anesthetics. In this case, temperature monitoring is used to detect either hyperthermia or hypothermia during surgery longer than 30 minutes.

- Transmissible febrile diseases. One of the most common symptoms of contagious diseases is fever. Furthermore, some patients at epidemic risk require isolation or quarantine, while keeping them closely monitored.

- Sepsis. Although fever is generally a central symptom in sepsis, it is not always present, and what is more, its absence is often a deleterious sign. The real core sign in sepsis, and more generally in the severe inflammatory response syndrome (SIRS), is tissue hypoperfusion. This can be monitored with a multichannel device, analyzing the temperature gradients. As described in [15], the study of temperature patterns, instead of absolute values, can be an early detector of sepsis and therefore facilitate a prompt antimicrobial therapy.

- Postoperative fevers. Fever is quite common after the first few days following a major surgery. It is necessary to provide a differential diagnosis of these fevers in order to rule out unexpected serious conditions.

- Neutropenia. It is common for neutropenic patients to become febrile. Because mortality rates associated with untreated bacterial infections in neutropenic patients are high, the rapid empiric treatment of these patients 
is critical. These patients require very close outpatient monitoring and assessment.

\section{Discussion}

At present, conventional fever monitoring is similar to the old technique of measuring the heart rate through the pulse every shift, and assuming this was all the information that could be obtained from the heart rhythm for clinical practice. Obviously, cardiac monitoring changed radically this scenario. We believe something similar can occur with temperature.

The conventional "disease-centred" thermometry is even more open to debate. The classical "fever patterns" (e.g. tertian, Pel-Ebstein, etc.) are no longer in use [16], and the area is ripe for new, more physiological approaches $[17,18]$. Furthermore, as a prototypical example of a complex system, it is an ideal field of interaction with the bourgeoning areas of Non Linear Dynamics and Fractal Geometry, in terms of signal processing schemes.

Moreover, the conventional idea of fever as a factual symptom overlooks most of the physiologic process. Building a fever is a painstaking, metabolically demanding activity, involving lypopolysacharides, macrophages, interleukins, hypothalamic set point modification, efferent circuits, etc. [20], that can arguably be detected before fever appears.

Conventional clinical practice only considers central temperature as medically relevant, whereas the real game is being played by the central/peripheral gradient. Continuous monitoring of both central and peripheral temperature could provide an indirect measure of the heat conservation / heat dissipation equilibrium.

Body temperature measurement faces a variety of challenges. There are many factors that need to be considered when making decisions related to the patient status, such as the body temperature distribution, the temperature baseline, ambient temperature, patient activity, and peripheral perfusion. The new approach described is able to aggregate all these factors in a single device. It can record peripheral, environmental, and core temperature readings simultaneously, and continuously, in an ambulatory manner, in contrast to current approaches. With such a tool, it will become possible to:

- Provide avenues for the development of new clinical procedures.

- Combine the existing knowledge of the underlying disease process with functional signaling data.

- Study how the thermoregulatory environment influences therapeutic response.

- Tailor treatments to individuals.

- Strengthen global public health surveillance to provide early warnings against emerging infectious diseases.

- Use new data sources and methods to improve global coverage, sensitivity, and timeliness. 


\section{Conclusion}

Body temperature has been an underrated physiological parameter. Despite its importance for a timely detection of hyper or hypo-thermia, it has been sparsely and inaccurately measured [9]. Furthermore, only absolute values at single locations have been taken into account. Current measurement devices were also focused in the traditional applications and uses of temperature readings, and did not contribute to a breakthrough in this field either.

We described in this paper a new device for a more advanced body temperature measurement and monitoring approach, including examples of already developed applications or prospective new uses. This device includes 4 channels for a multi-site measurement, including ambient temperature recording. Beyond the classical approach, multiple channels enable the study of temperature data at different locations, such as skin and core temperatures, peripheral perfusion, gradient or inversion studies, among many more different novel and clinically powerful applications. Such a non-invasive device can contribute to a higher degree of clinical certainty, providing a more consistent evidence about the physiological patient's status.

There are a myriad of advanced applications that can benefit from the temperature monitoring proposed in this paper. Some of them have already been tested, such as fever peak detection, pre-febrile profiling, or patient prognosis. Others can also be addressed seamlessly, since the features and capabilities match their requirements, such as neutropenic patient monitoring, study fevers of unknown origin, thermal ablation, orthopedic surgery, or patient at epidemic risk, isolated, or in quarantine, control. This device can become a game-changer in clinical thermometry and no stone should be left unturned in terms of possible new applications [21].

This device has been CE marked, is patent pending, and the current manufacturer, the company Innovatec Sensing \& Communications (www.innovatecsc.com), has obtained the corresponding ISO-13485 certification. Its accuracy has also been certified by an independent metrology body.

\section{References}

1. Gai M., Merlo I., Dellepiane S., Cantaluppi V., Leonardi G., Fop F., Guarena C., Grassi G., Biancore L., Glycemic pattern in diabetic patients on hemodialysis: Continuous Glucose Monitoring (CGM) analysis, Blood Purif., 38(1), 68-73, (2014)

2. Kondziella D., Friberg C.K., Wellwood I., Reiffurth C., Fabricius M., Dreier J.P., Continuous EEG monitoring in aneurysmal subarachnoid hemorrhage: A systematic review, Neurocrit. Care, (2014)

3. Ciccone A., Celani M.G., Chiaramonte R., Rossi C., Righetti E., Continuous versus intermittent physiological monitoring for acute stroke, Cochrane Database Syst. Rev., 31 (2013)

4. Kushimoto S., Yamanouchi S., Endo T., Sato T., Nomura R. Fujita M., Kudo D., Omura T., Miyagawa N., Sato T., Body temperature abnormalities in non-neurological critically ill patients: A review of the literature, Journal of Intensive Care, 2, (2014)

5. Mc Callum L., Higgings D., Measuring body temperature, Nursing Times, 108, 20-22 (2012) 
6. Varela M., Ruiz-Esteban R., Martinez-Nicolas A., Cuervo-Arango A., Barros C., Delgado E.G., Catching the spike and tracking the flow: Holter-temperature monitoring in patients admitted in a general internal medicine ward, Int. J. of Clinical Practice, 65, 12, 1283-1288, (2011)

7. Lopes F., Peres D., Bross A., Melot C., Vincent J.L., Serial evaluation of the SOFA score to predict outcome in critically ill patients, J. of the American Medical Association, 286, 1754-1758, (2001)

8. Vincent J.L., Moreno R., Clinical review: Scoring systems in the critically ill, Critical Care, 14, (2010)

9. Sund-Levander M., Grodzinsky E., Time for a change to assess and evaluate body temperature in clinical practice, International Journal of Nursing Practice, 15, 241-249, (2009)

10. Cuesta-Frau D., Varela M., Aboy M., Miro P., Description of a portable wireless device for body temperature acquisition and analysis, Sensors, 9(10), 7648-7663, (2009)

11. Varela M., Cuesta-Frau D., Madrid J.A., Churruca J., Miro-Matinez P., Ruiz R., Marinez C., Holter monitoring of central and peripheral temperature: Possible uses and feasibility study in outpatient settings, Journal of Clinical Monitoring and Computing, 4(23), 209-216, (2009)

12. Jordan J., Miro P., Cuesta-Frau D., Varela M., Vargas B., Aplicacion de analisis multivariante para la deteccion de estados prefebriles en pacientes ingresados (in Spanish), XXXIV Congreso Nacional de Estadistica e Investigacion Operativa, Castellon(Spain), (2013)

13. Richman, J.; Moorman, J.R.; Physiological time-series analysis using Approximate Entropy and Sample Entropy, Am. J. Physiol. Heart Circ. Physiol., 2000, 278 (6), H20392049.

14. Young P., Saxena M., Eastwood G.M., Bellomo R., Beasley R., Fever and fever management among intensive care patients with known or suspected infection: A multicentre prospective cohort study, Crit. Care Resusc., 13, 97-102, (2011)

15. Drewry A.M., Fuller B.M., Bailey T.C., Hotchkiss R.S., Body temperature patterns as a predictor of hospital-acquired sepsis in afebrile adult intensive care unit patients: a case-control study, Crit. Care, 17, (2013)

16. Musher D., Fainstein V., Young E., Pruett T., Fever patterns. Their lack of significance, Arch. Intern. Med., 139(11):1225-8, (1979)

17. Varela M., Calvo M., Chana M., Gomez-Mestre I., Asensio R., Galdos P., Clinical implications of temperature curve complexity in critically ill patients, Crit. Care Med., 33(12), 2764-2771, (2005)

18. Varela M., Churruca J., Gonzalez A., Martin A., Ode J., Galdos P., Temperature curve complexity predicts survival in critically ill patients, Am. J. Respir. Crit. Care Med., 174(3), 290-298, (2006)

19. Cuesta-Frau D., Varela M., Miro P., Galdos P., Abasolo D., Hornero R., Aboy M., Predicting survival in critical patients by use of body temperature regularity measurement based on Approximate Entropy, Med. Biol. Eng. Computing, 45, 671-678, (2007)

20. Mackiowak P., Temperature regulation and the pathogenesis of fever, Principles and Practice of Infectious Diseases, 765-778, Churchill Livingston Elsevier, New York, (2010)

21. Cherbuin N., Brinkman C., Cognition is cool: Can hemispheric activation be assessed by tympanic membrane thermometry?, Brain and Cognition, 54, 228-231, (2004) 